\title{
ON A COMPLEMENTARITY PROBLEM ASSOCIATED WITH NONDIFFERENTIABLE PROGRAMMING
}

\author{
B. D. CRAVEN 1 , B. MOND ${ }^{2}$ and J. PARIDA ${ }^{3}$
}

(Received 8 September 1981; revised 27 October 1982)

\begin{abstract}
This paper deals with the question of the existence of a solution to the stationary-point problem corresponding to a given nonlinear nondifferentiable program. An existence theorem for the stationary-point problem is presented under some convexity and regularity conditions on the functions involved, which also guarantee an optimal solution to the nonlınear program.
\end{abstract}

\section{Introduction}

A mathematical programming problem with an objective function containing the square root of a positive semidefinite quadratic form has been discussed by many authors (see references in [5]). Sinha [11,12] has shown that in the formulation of a deterministic model of the stochastic linear programming problem, the objective function contained a term of the above form. Mond [5] has generalized the formulation in [12], and derived necessary and sufficient conditions for optimality for such programming problems. Since the usual methods for solving this type of problem are not applicable because of the nondifferentiability of the objective function, it is solved by solving the corresponding dual problems. For this purpose, it is always assumed that the primal has an optimal solution. One feels it useful if the existence of an optimal solution to the problem is ensured before the

\footnotetext{
'Department of Mathematics, University of Melbourne, Parkville, Victoria 3052.

${ }^{2}$ Department of Mathematics, La Trobe University, Bundoora, Victoria 3083.

${ }^{3}$ Department of Mathematics, Regional Engineering College, Rourkela 769008 , India. (The contribution of this author was made while he was a visitor to the University of Melbourne.)

(c) Copyright Australian Mathematical Society 1984, Serial-fee code 0334-2700/84
} 
actual computation is taken up. With this point in view, consider the programming problem:

$$
\begin{gathered}
(\mathrm{P}): \text { Minimize } F(x) \equiv f(x)+\sum_{J=1}^{p}\left(x^{T} B_{J} x\right)^{1 / 2} \\
\text { subject to } x \in X,
\end{gathered}
$$

where $X=\left\{x \in X^{0}: x \geqslant 0, g(x) \geqslant 0\right\}, X^{0}$ is an open set in $R^{n}, f: Y^{0} \rightarrow R$ and $g$ : $X^{0} \rightarrow R^{m}$ are differentiable functions on $X^{0}$, and $B_{j}, j=1, \ldots, p$, is an $n \times n$ symmetric positive semidefinite matrix. A test will be obtained for the existence of an optimal solution. This is primarily of interest when the feasible set $X$ is unbounded, since an optimal solution will always exist if $X$ is bounded, and hence compact. The results may also be of use in cases when it is not easy to determine whether or not the feasible set $X$ is bounded.

Following Mond [5], a stationary-point problem for $(\mathrm{P})$, very similar to the Kuhn-Tucker stationary-point problem [4] for the usual nonlinear differentiable program is given as follows: Let $J=\{1,2, \ldots, p\}$, and for $x \in X$, let $K(x)=\{j$ $\left.\in J: B_{j} x=0\right\}$.

(CP): Find $x \in R^{n}, u \in R^{m}, w_{j} \in R^{n}, j \in K(x)$, such that

$$
\begin{aligned}
& \chi\left(x, u, w_{J}\right) \equiv \nabla \phi(x, u)+\sum_{j \in J \backslash K(x)} B_{j} x /\left(x^{T} B_{J} x\right)^{1 / 2} \\
& +\sum_{j \in K(x)} B_{j} w_{j} \geqslant 0, \\
& g(x) \geqslant 0, \\
& 1-w_{j}^{T} B_{j} w_{J} \geqslant 0, \quad j \in K(x), \\
& x \geqslant 0, \quad u \geqslant 0, \\
& \left\langle x\left(x, u, w_{j}\right), x\right\rangle=0, \quad\langle g(x), u\rangle=0,
\end{aligned}
$$

where $\phi(x, u)=f(x)-u^{T} g(x), \quad \nabla \phi(x, u)=\nabla f(x)-A(x) u, \quad \nabla$ denotes the gradient with respect to $x$ and $A(x)$ is the Jacobian matrix of the mapping $g$ defined by $A_{\jmath}(x)=\partial g_{\jmath} / \partial x_{1}$. It is easy to check that if $\left(x, u, w_{j}\right)$ is a solution to (CP) and if $f$ is convex and $g$ is concave at $x$, with respect to $X$ (see [4], definition 4.1.1), then $x$ is an optimal solution of (P). Thus for $f$ convex and $g$ concave, (1.1) is a sufficient condition for $x$ to be optimal for $(\mathrm{P})$.

Given a function $M: R_{+}^{n} \rightarrow R^{n}$, the problem of finding an $x \in R^{n}$ such that

$$
x \geqslant 0, \quad M(x) \geqslant 0, \quad\langle M(x), x\rangle=0,
$$

is known as the complementarity problem. The Kuhn-Tucker stationary-point problem for the usual nonlinear differentiable program with nonnegative variables takes the form (1.2). Hence, the problem of the existence of a solution to the nonlinear program can be reduced to a problem of the form (1.2) (see [1], [6] and 
[2]). The stationary-point problem for (P) does not take exactly the form (1.2), and thus, the problem (1.1) is not a complementarity problem in the true sense of the term. In order to maintain similarity, we call (1.1) the (CP) associated with (P) and study the question of the existence of an optimal solution to $(\mathrm{P})$ via the $(\mathrm{CP})$. Convexity of a function is defined in terms of its directional derivative and an existence theorem for (CP) is established under some conditions on $f$ and $g$ which also guarantee the existence of an optimal solution to (P).

\section{Preliminaries}

Throughout this paper, $\Gamma$ denotes an index set and $R^{n}$ denotes $n$-dimensional Euclidean space with the usual inner product $\langle x, y\rangle$ of $x, y \in R^{n}$. Further, $R_{+}$ and $R_{+}^{n}$ denote the set of nonnegative numbers and the nonnegative orthant, $\left\{x \in R^{n}: x \geqslant 0\right\}$ of $R^{n}$, respectively. Finally, $e \in R^{n}$ denotes a vector all of whose components are unity.

For a function $H: X^{0} \rightarrow R$, a point $x \in X^{0}$ and a vector $z \in R^{n}$, the directional derivative of $H$ at $x$ in the direction of $z$, which we denote by $H^{\prime}(x ; z)$, is given by

$$
H^{\prime}(x ; z)=\lim _{\tau \rightarrow 0+}[H(x+\tau z)-H(x)] / \tau,
$$

where $\tau \rightarrow 0+$ means $\tau$ approaches zero through positive values. If $H$ is differentiable at $x$, then $H^{\prime}(x ; z)=[\nabla H(x)]^{T} z$. It has been shown by Mond and Schechter [7] that, for the function $H(x)=f(x)+\left(x^{T} B x\right)^{1 / 2}$,

$$
\begin{gathered}
H^{\prime}(x ; z)=z^{T} \nabla f(x)+z^{T} B x /\left(x^{T} B x\right)^{1 / 2} \quad \text { if } x^{T} B x>0, \\
H^{\prime}(x ; z)=z^{T} \nabla f(x)+\left(z^{T} \dot{B} z\right)^{1 / 2} \text { if } x^{T} B x=0 .
\end{gathered}
$$

This result is easily extended to the objective function $F(x)=f(x)+$ $\sum_{j=1}^{p}\left(x^{T} B_{J} x\right)^{1 / 2}$, and we have

$$
\begin{aligned}
F^{\prime}(x ; z)= & z^{T} \nabla f(x)+\sum_{j \in J \backslash K(x)} z^{T} B_{j} x /\left(x^{T} B_{j} x\right)^{1 / 2} \\
& +\sum_{j \in K(x)}\left(z^{T} B_{j} z\right)^{1 / 2} .
\end{aligned}
$$

A function $H: X^{0} \rightarrow R$ is said to be convex at $x^{0} \in X^{0}$ with respect to $X^{0}$ if, for each $x \in X^{0}, H^{\prime}\left(x^{0} ; x-x^{0}\right)$ exists and

$$
H(x)-H\left(x^{0}\right)-H^{\prime}\left(x^{0} ; x-x^{0}\right) \geqslant 0 .
$$

$H$ is said to be convex on $X^{0}$ if it is convex at each $x \in X^{0} . H$ is concave at $x^{0} \in X^{0}$ (concave on $X^{0}$ ) if and only if $-H$ is convex at $x^{0}$ (convex on $X^{0}$ ). 
A function $H: X^{0} \rightarrow R$ is said to be pseudoconvex at $x^{0} \in X^{0}$ with respect to $X^{0}$ if, for each $x \in X^{0}, H^{\prime}\left(x^{0} ; x-x^{0}\right)$ exists and

$$
H^{\prime}\left(x^{0} ; x-x^{0}\right) \geqslant 0 \Rightarrow H(x) \geqslant H\left(x^{0}\right) .
$$

$H$ is said to be pseudoconvex on $X^{0}$ if it is pseudoconvex at each $x \in X^{0} . H$ is pseudoconcave at $x^{0}$ (on $X^{0}$ ) if and only if $-H$ is pseudoconvex at $x^{0}$ (on $X^{0}$ ).

A function $H: X^{0} \rightarrow R$ is said to be quasiconcave at $x^{0} \in X^{0}$ with respect to $\bar{X}^{\hat{0}}$ if, for each $x \in X^{\hat{0}}, H^{\prime}\left(x^{\hat{0}} ; x-x^{\hat{0}}\right)$ exists and

$$
H(x) \geqslant H\left(x^{0}\right) \Rightarrow H^{\prime}\left(x^{0} ; x-x^{0}\right) \geqslant 0 .
$$

$H$ is said to be quasiconcave on $X^{0}$ with respect to $X^{0}$ if it is quasiconcave at each $x \in X^{0}$.

The concept of convexity at a point of a set (on a set) with respect to the same set to which the point under consideration belongs is introduced by Mangasarian [4]. Note that the above concept of pseudoconvexity in terms of directional derivative generalizes the concept of pseudoconvexity given in [4].

For $x \in X$, define the set $Z_{x}$ by

$$
Z_{x}=\left\{z: z^{T} \nabla g_{1}(x) \geqslant 0\left(\forall i \in Q_{1}\right), z_{j} \geqslant 0\left(\forall j \in Q_{2}\right) \text { and } F^{\prime}(x ; z)<0\right\}
$$

where

$$
Q_{1}=\left\{i: g_{1}(x)=0\right\} \text { and } Q_{2}=\left\{j: x_{\jmath}=0\right\} .
$$

If $x$ is an optimal solution of (P) and $Z_{x}$ is empty, then (1.1) is a necessary condition for optimality. This follows from a theorem of Sinha [12, page 380]. As observed by Mond and Schechter [7], the condition that $Z_{x}$ is empty at an optimal solution of $(\mathrm{P})$ is implied by the Slater constraint qualification.

Consider the following conditions involving the functions $f, g$ and the matrices $B$.

CONDITION 1. The function $g$ is pseudoconcave on $R_{+}^{n}$, and there exists an $\hat{x} \in X$ such that $g_{1}(\hat{x})>0$ whenever $g_{1}$ is non-affine.

CONDITION 2. The point $\bar{x} \in X$ satisfies the inequality

$$
q(\bar{x}) \equiv \nabla f(\bar{x})+\sum_{J \in J \backslash K(\bar{x})} B j \bar{x} /\left(\bar{x}^{T} B_{j} \bar{x}\right)^{1 / 2}>0 .
$$

The proof of the following lemma is omitted, since it follows by making some minor modifications to the proof of Mond and Schechter [7, page 613].

Lemma 1. Assume that Condition 1 is satisfied. If $\bar{x}$ is an optimal solution of (P), then $Z_{\bar{x}}$ is empty. 


\section{Main results}

In the discussion to follow, we always assume that the feasible set $X$ of $(\mathrm{P})$ is nonempty. Define the set $X(\alpha) \subset X$ by

$$
X(\alpha)=\left\{x: x \in X, e^{T} x \leqslant \alpha\right\}
$$

for every real $\alpha>0$. Since $X$ is nonempty, there exists an $\alpha_{0}>0$ such that $X(\alpha)$ is nonempty for every $\alpha_{0} \leqslant \alpha<\infty$.

The following lemma gives a necessary condition for (CP) not to have a solution.

Lemma 2. Assume that Condition 1 is satisfied. If (CP) has no solution then the following holds: There exist sequences $\left\{x^{t}\right\}_{1 \in \Gamma}$ and $\left\{u^{i}\right\}_{i \in \Gamma}$, both corresponding to an infinite sequence $\left\{\alpha_{i}\right\}_{i \in \Gamma}$ with $\alpha_{t} \rightarrow \infty$, such that

$$
\left\{x^{i} / \alpha_{1}\right\}_{, \in \Gamma} \text { converges to a nonzero vector } t \geqslant 0,
$$

and for every $i \in \Gamma$,

$$
\begin{gathered}
x^{i} \in X\left(\alpha_{1}\right), \quad u^{i} \geqslant 0, \quad\left\langle g\left(x^{\prime}\right), u^{i}\right\rangle=0, \\
F^{\prime}\left(x^{i} ; x-x^{i}\right)-\left\langle A\left(x^{i}\right) u^{i}, x-x^{i}\right\rangle \geqslant 0 \quad \text { for every } x \in X\left(\alpha_{i}\right) .
\end{gathered}
$$

Proof. The proof $\hat{x}$ given by Condition 1 satisfies $\hat{x} \in X\left(\alpha^{*}\right)$, for some sufficiently large $\alpha^{*}$. Let $\alpha_{0}^{\prime}=\max \left(\alpha_{0}, \alpha^{*}\right)$. The sets $X(\alpha)$, for $\alpha_{0}^{\prime} \leqslant \alpha<\infty$, are compact. The continuous function $F$ attains its infimum on each such compact set $X(\alpha)$. Let $x^{\alpha}$ be the minimal point of $F$ with respect to $X(\alpha)$ for $\alpha_{0}^{\prime} \leqslant \alpha<\infty$. By hypothesis and Lemma 1 , we obtain, as a necessary condition of optimality, vectors $u^{\alpha}, w_{j}^{\alpha}, j \in K\left(x^{\alpha}\right)$, and a scalar $\xi^{\alpha}$, all corresponding to $x^{\alpha}$, such that

$$
\begin{aligned}
& x^{\alpha} \geqslant 0, \quad g\left(x^{\alpha}\right) \geqslant 0, \\
& u^{\alpha} \geqslant 0, \quad\left\langle g\left(x^{\alpha}\right), u^{\alpha}\right\rangle=0, \\
& \chi\left(x^{\alpha}, u^{\alpha}, w_{j}^{\alpha}\right)+\xi^{\alpha} e \geqslant 0, \\
& \left\langle\chi\left(x^{\alpha}, u^{\alpha}, w_{j}^{\alpha}\right)+\xi^{\alpha} e, x^{\alpha}\right\rangle=0, \\
& w_{j}^{\alpha^{T}} B, w_{j}^{\alpha} \leqslant 1 \quad \text { for } j \in K\left(x^{\alpha}\right) \text {, } \\
& e^{T} x^{\alpha} \leqslant \alpha, \quad \xi^{\alpha} \geqslant 0, \quad \xi^{\alpha}\left(\alpha-e^{T} x^{\alpha}\right)=0 .
\end{aligned}
$$

If there is an $\alpha$ such that $\xi^{\alpha}=0$, then $\left(x^{\alpha}, u^{\alpha}, w_{f}^{\alpha}\right)$ is a solution to (CP). Therefore, we conclude that if (CP) has no solution, then $\xi^{\alpha}>0$ for every $\alpha_{0}^{\prime} \leqslant \alpha<\infty$. Now by (3.9) we have $e^{T} x^{\alpha}=\alpha$. Let $t^{\alpha}=x^{\alpha} / \alpha$, and then $t^{\alpha} \geqslant 0$ and $e^{T_{t}}{ }^{\alpha}=1$. This shows that the set of points $t^{\alpha}$ lies in a compact set, and hence, there exists a convergent sequence of $t^{\alpha}$ with $\alpha \rightarrow \infty$. Let this sequence of $t^{\alpha}$ 
correspond to the sequence $\left\{\alpha_{i}\right\}_{t \in \Gamma}$. Let $t$ be the limit of this sequence. Thus we obtain a vector

$$
t=\lim _{i \in \Gamma} t^{t}=\lim _{i \in \Gamma}\left(x^{t} / \alpha_{t}\right),
$$

where $t \geqslant 0$ and $e^{T} t=1$. Clearly, $t \neq 0$. This establishes (3.1) and (3.2). Further, for any $x \geqslant 0$, we obtain from (3.6) and (3.7) that

$$
\left\langle x\left(x^{\prime}, u^{i}, w_{j}^{\prime}\right), x-x^{i}\right\rangle+\xi^{\prime} e^{T}\left(x-x^{\prime}\right) \geqslant 0
$$

for every $i \in \Gamma$. But $e^{T}\left(x-x^{\prime}\right)=e^{T} x-\alpha_{i} \leqslant 0$ for every $i \in \Gamma$ if $x \in X\left(\alpha_{t}\right)$. From the generalized Schwarz inequality [9, page 262] and (3.8), we have

$$
\begin{aligned}
\left\langle\sum_{, \in K\left(x^{\prime}\right)} B_{j} w_{j}^{\prime}, x-x^{\prime}\right\rangle & \leqslant \sum_{j \in K\left(x^{\prime}\right)}\left(w_{j}^{i} B_{j} w_{\jmath}^{\prime}\right)^{1 / 2}\left[\left(x-x^{\prime}\right)^{T} B_{j}\left(x-x^{\prime}\right)\right]^{1 / 2} \\
& \leqslant \sum_{j \in K\left(x^{l}\right)}\left[\left(x-x^{\prime}\right)^{T} B_{j}\left(x-x^{\prime}\right)\right]^{1 / 2} .
\end{aligned}
$$

Hence, it follows from (3.10) and the definition of $F^{\prime}$ as given by (2.1) that

$$
F^{\prime}\left(x^{i} ; x-x^{i}\right)-\left\langle A\left(x^{i}\right) u^{\prime}, x-x^{i}\right\rangle \geqslant 0 \text { for every } x \in X\left(\alpha_{t}\right) .
$$

This completes the proof of the lemma.

We define the following sets.

$$
\begin{aligned}
& V=\left\{(x, u): x \in X, u \in R_{+}^{m},\right. \\
&\left.F^{\prime}(x ; y-x)-\langle A(x) u, y-x\rangle \geqslant 0 \text { for some } y(\neq x) \in X\right\}, \\
& U=\left\{u \in R_{+}^{m}:(x, u) \in V \text { for some } x \in X\right\}, \text { and } \\
& \quad X_{u}=\{x:(x, u) \in V\} \text { for } u \in U .
\end{aligned}
$$

Obviously, $X_{u} \subset X$. We note that if $(x, u) \in V$ then $u \in U$ and $x \in X_{u}$.

LEMMA 3. (a) If $\left(x, u, w_{j}\right)$ is a solution to (CP), and $X \neq\{x\}$, then

$$
F^{\prime}(x ; y-x)-\langle A(x) u, y-x\rangle \geqslant 0 \text { for all } y \in X,
$$

and hence, if there exists $y(\neq x) \in X$, then $u \in U$ and $x \in X_{u}$.

(b) Assume that $X$ is unbounded. If, for some $\bar{x} \in X$,

$$
q(\bar{x})=\nabla f(\bar{x})+\sum_{j \in J \backslash K(\bar{x})} B_{j} \bar{x} /\left(\bar{x}^{T} B_{j} \bar{x}\right)^{1 / 2}>0,
$$

then $0 \in U$ and $\bar{x} \in X_{u}$ with $u=0$.

(c) Assume that $g$ is pseudoconcave (or quasiconcave) on $R_{+}^{n}$. If there exist $u \in R_{+}^{m}, x \in X$ such that $\langle g(x), u\rangle=0$, then $\langle A(x) u, y-x\rangle \geqslant 0$ for all $y \in X$. 
Proof. (a) follows from (1.1) and the inequality

$$
\left\langle\sum_{J \in K(x)} B_{j} w_{J}, y-x\right\rangle \leqslant \sum_{, \in K(x)}\left[(y-x)^{T} B_{J}(y-x)\right]^{1 / 2} .
$$

(b) Since $X$ is unbounded, there is a sequence $\left\{x^{p}\right\}$ in $X$ such that $\left\|x^{p}\right\|_{\infty} \rightarrow \infty$, where $\|x\|_{\infty}=\max \left\{\left|x_{i}\right|: 1 \leqslant i \leqslant n\right\}$. Note that for $x \in X,\left|x_{i}\right|=x_{i}$ for every $i$. Let $\|\bar{x}\|_{\infty}=\beta$, and denote $\left\|x^{p}\right\|$ by $\mu(p)$. Then $\mu(p) \rightarrow \infty$ as $p \rightarrow \infty$. It follows from this that

$$
\begin{aligned}
\left\langle q(\bar{x}), x^{p}-\bar{x}\right\rangle & =\sum_{i=1}^{n} q_{i}(\bar{x})\left(x_{i}^{p}-\bar{x}_{i}\right) \\
& \geqslant\left\|x^{p}\right\|_{\infty}\left(\min _{t} q_{i}(\bar{x})\right)-n\|\bar{x}\|_{\infty}\left(\max _{t} q_{i}(\bar{x})\right) \\
& =\mu(p)\left(\min _{t} q_{t}(\bar{x})\right)-n \beta\left(\max _{t} q_{i}(\bar{x})\right) \\
& \geqslant 0
\end{aligned}
$$

if $p$ is taken sufficiently large. Let one such value of $p$ be $p_{1}$ for which the inequality holds. Now, by taking $y=x^{p_{1}}$, we have $\langle q(\bar{x}), y-\bar{x}\rangle \geqslant 0$. Since $B_{J}$, $j \in K(\bar{x})$, is positive semidefinite,

$$
\sum_{j \in K(\bar{x})}\left[(y-\bar{x})^{T} B_{J}(y-\bar{x})\right]^{1 / 2} \geqslant 0 .
$$

Hence, we have $F^{\prime}(\bar{x} ; y-\bar{x}) \geqslant 0$, which implies $(\bar{x}, 0) \in V$.

(c) By the hypothesis, we have

$$
\sum_{i=1}^{m} u_{i} g_{t}(y) \geqslant 0 \text { and } \sum_{i=1}^{m} u_{t} g_{i}(x)=0
$$

where $u_{t} \geqslant 0, g_{\imath}(y) \geqslant 0$ and $g_{t}(x) \geqslant 0$ for each $i$. This implies that

$$
u_{i} g_{\imath}(y) \geqslant 0=u_{\imath} g_{i}(x) \text { for each } i \text {. }
$$

Let $I_{0}=\left\{i: u_{t}=0\right\}$. Then for $i \notin I_{0}$, we have $g_{t}(y) \geqslant g_{t}(x)$. If $g_{l}$ is pseudoconcave on the convex set $R_{+}^{n}$, it is quasiconcave [4], which implies $\left\langle\nabla g_{1}(x), y-\right.$ $x\rangle \geqslant 0$. Now multiplying by $u_{t}$, we obtain

$$
\left\langle u_{\imath} \nabla g_{1}(x), y-x\right\rangle \geqslant 0 \quad \text { for each } i \notin I_{0} .
$$

Since $u_{i}=0$ for $i \in I_{0}$, it follows by taking the sum over $i$ that $\langle A(x) u, y-x\rangle \geqslant$ 0 .

THEOREM 1. For problem (P), assume both the constraint qualification (Condition 1 ), and also Condition 2, that $q(\bar{x})>0$ for some $\bar{x} \in X$. If, for each fixed $u \in U, F$ is pseudoconvex in the variable $x$ on the set $X_{u}$ with respect to $X$, then (CP) has a solution, and hence, (P) has an optimal solution. 
Proof. If (CP) has a solution, then by the convexity assumption, $(\mathrm{P})$ has an optimal solution. This can be seen as follows. Let $\left(x, u, w_{j}\right)$ be a solution to (CP). Hence, if $x$ is not the only feasible solution of (P), by Lemmas 3(a) and 3(c) we have

$$
F^{\prime}(x ; y-x) \geqslant 0 \text { for all } y \in X,
$$

which by the pseudoconvexity of $F$ yields $F(y) \geqslant F(x)$ for all $y \in X$.

We show that (CP) has a solution by contradiction. Only the case of $X$ unbounded need be considered, since (P) has always an optimal solution when $X$ is bounded. Assuming that (CP) has no solution, by Lemma 2 we have a sequence $\left\{\alpha_{i}\right\}_{t \in \Gamma} \subset R_{+}$with $\alpha_{\imath} \rightarrow \infty$, corresponding to which there exist sequences $\left\{x^{t}\right\}_{t \in \Gamma}$ and $\left\{u^{i}\right\}_{i \in \Gamma}$ such that, for each $i \in \Gamma$,

$$
\begin{gathered}
x^{i} \in X\left(\alpha_{1}\right), \quad u^{\prime} \in R_{+}^{m}, \quad\left\langle g\left(x^{\prime}\right), u^{i}\right\rangle=0, \\
F^{\prime}\left(x^{i} ; x-x^{i}\right)-\left\langle A\left(x^{\prime}\right) u^{i}, x-x^{\prime}\right\rangle \geqslant 0 \quad \text { for all } x \in X\left(\alpha_{\imath}\right) .
\end{gathered}
$$

For some $s \in \Gamma$, the points $\hat{x}$ and $\bar{x}$ given by Conditions 1 and 2 satisfy $e^{T} \hat{x} \leqslant \alpha_{\text {, }}$ and $e^{r} \bar{x} \leqslant \alpha_{1}$ for every $\alpha_{l} \geqslant \alpha_{s}, i \in \Gamma$. Then we have

$$
F^{\prime}\left(x^{\prime} ; \bar{x}-x^{\prime}\right)-\left\langle A\left(x^{\prime}\right) u^{i}, \bar{x}-x^{\prime}\right\rangle \geqslant 0 \text { for all } i \in \Gamma \text { with } \alpha_{l} \geqslant \alpha_{s} .
$$

By the pseudoconvexity assumption and Lemma 3(c), this implies

$$
F(\bar{x}) \geqslant F\left(x^{t}\right) \text {. }
$$

We thus have

$$
F(\bar{x}) \geqslant F\left(x^{\prime}\right) \text { for every } i \in \Gamma \text { with } \alpha_{t} \geqslant \alpha_{s} .
$$

We now distinguish between two cases.

Case 1: $F(\bar{x})=F\left(x^{\prime}\right)$ for every $i \in \Gamma$ with $\alpha_{1} \geqslant \alpha_{s}$. This implies that $F(\bar{x})$ is the minimum value of $F$ over $X$ and (CP) has a solution.

Case 2: $F(\bar{x})>F\left(x^{r}\right)$ for some $r \in \Gamma$ with $\alpha_{r} \geqslant \alpha_{s}$. Since $F\left(x^{\prime}\right)$ does not increase with increasing $\alpha_{t}$, it is clear that $F(\bar{x})>F\left(x^{i}\right)$ for every $i \in \Gamma$ with $\alpha_{t} \geqslant \alpha_{r} \geqslant \alpha_{s}$. By Lemma 3(b), we have $0 \in U$ and $\bar{x} \in X_{u}$ with $u=0$, which implies that $F$ is pseudoconvex at $\bar{x}$. But by the pseudoconvexity of $F, F(\bar{x})>$ $F\left(x^{\prime}\right)$ implies $F^{\prime}\left(\bar{x} ; x^{\prime}-\bar{x}\right)<0$. Thus we have $F^{\prime}\left(\bar{x} ; x^{\prime}-\bar{x}\right)<0$ for every $i \in \Gamma$ with $\alpha_{t} \geqslant \alpha_{r} \geqslant \alpha_{s}$. Now dividing by $\alpha_{t}$ and taking the limit, this yields

$$
t^{T} \nabla f(\bar{x})+\sum_{J \in J \backslash K(\bar{x})} t^{T} B_{j} \bar{x} /\left(\bar{x}^{T} B_{j} \bar{x}\right)^{1 / 2}+\sum_{J \in K(\bar{x})}\left(t^{T} B_{j} t\right)^{1 / 2} \leqslant 0 .
$$

This is a contradiction since $(0 \neq) t \geqslant 0$. 
Corollary 1. Assume that Conditions 1 and 2 are satisfied. If $f$ is convex on $X$ with respect to $X$, then there exists a solution to (CP) and hence an optimal solution to $(\mathrm{P})$.

REMARK. Note that a necessary condition for $x^{0} \in X$ to be an optimal solution of $(\mathrm{P})$ is that there exist some $u^{0} \in R_{+}^{m}$ satisfying

$$
\begin{gathered}
F^{\prime}\left(x^{0} ; y-x^{0}\right)-\left\langle A(x) u^{0}, y-x^{0}\right\rangle \geqslant 0 \quad \text { for all } y \in X, \\
\left\langle g\left(x^{0}\right), u^{0}\right\rangle=0 .
\end{gathered}
$$

This condition (implied by (1.1)) is sufficient when some convexity condition is assumed. Let $Q_{o}=\left\{i: g_{l}\left(x^{0}\right)=0\right\}$. If there exist $x^{0} \in X, u^{0} \in R_{+}^{m}$ satisfying (3.11), and if $f$ is convex at $x^{0}$ with respect to $X$ [or $F$ is pseudoconvex at $x^{0}$ with respect to $X]$, and if $g_{1}, i \in Q_{0}$ [or $u_{1}^{0} g_{1}, i \in Q_{0}$ ] are quasiconcave at $x^{0}$ with respect to $X$, then it follows that $x^{0}$ is an optimal solution of (P). Sufficiency conditions of this sort can be found for $f$ convex and $g$ concave in [5] and for $f$ convex and $g$ quasiconcave in [10].

Condition (3.11) holds if (CP) has a solution. In Theorem 1, a solution to (CP) is proved to exist, under a stronger convexity condition, which while making (3.11) sufficient for optimality, ensures that a solution to (CP) exists. In Lemma 2, necessary conditions are obtained for a minimal point, $x_{\alpha}$ of $F$ over $X(\alpha)$, using the Slater Condition, which does not depend on the particular point $x_{\alpha}$. Analogous results hold with other constraint qualifications that are applicable to the sequence of programs obtained by substituting $X(\alpha)$ for $X$. In such cases, it is possible to weaken the requirements on the function $g$ to quasiconcavity.

So far as the authors know, there are no existence results previously known for nondifferentiable programming. For the differentiable problem,

(PD): Minimize $f(x)$ subject to $x \in X$, obtained by setting each $B_{J}=0$ in (P), the sufficient conditions for optimality in [3, Theorems 1.1.1-1.1.4] are comparable to those for (P) discussed above. Kojima [2] showed that an optimal solution exists for (PD) if $X$ is bounded, a Slater condition holds, and $f$ is convex and $g$ concave; but $X$ bounded closed and $f$ continuous suffice. Parida and Sahoo [8] obtained an existence result for (PD) assuming $f$ convex and $g$ concave on $R_{+}^{\prime \prime}$, and there exists $\bar{x} \geqslant 0$ with $g(\bar{x})>0, \nabla f(\bar{x})>0$. This is a special case of Corollary 1. Some minor changes in the proof of Lemma 2 and Theorem 1 prove the following, more general, existence result.

THEOREM 2. Suppose that $f$ is pseudoconvex on $X$ with respect $t o x$ and $g$ is pseudoconcave on $R_{+}^{n}$. If there exists feasible $\bar{x}$ such that $g_{i}(\bar{x})>0$ for all non-affine $g_{1}$ and $\nabla f(\bar{x})>0$, then (PD) possesses an optimal solution. 
In order to apply the sufficient criterion of Theorem 1, all that is needed is one feasible point $\bar{x}$ (often from an infinite number of such points), for which $q(\bar{x})>0$; or, for Theorem 2, for which $\bar{x} \geqslant 0, g(\bar{x})>0, \nabla f(\bar{x})>0$. Such a point may often be easily found, as in examples (A), (D) and (E) below. However, examples (B) and (C) show that a minimum may be attained, without necessarily having $q(\bar{x})>0$ for any feasible $\bar{x}$. Example (F) shows that the sufficient hypothesis $q(\bar{x})>0$ cannot be weakened to $q(\bar{x}) \geqslant 0$.

Example (A) Minimize

$$
\begin{aligned}
F(x) & =x_{1}^{2}+2 x_{2}^{2}+\left\{\left[x_{1} x_{2}\right]\left[\begin{array}{ll}
1 & 0 \\
0 & 1
\end{array}\right]\left[\begin{array}{l}
x_{1} \\
x_{2}
\end{array}\right]\right\}^{1 / 2} \\
& =x_{1}^{2}+2 x_{2}^{2}+\left(x_{1}^{2}+x_{2}^{2}\right)^{1 / 2}
\end{aligned}
$$

subject to $g_{1}(x)=x_{1}-1 \geqslant 0, g_{2}(x)=-e^{-x_{2}}+1 \geqslant 0, x_{1} \geqslant 0, x_{2} \geqslant 0$.

Here $F$ is convex and the constraint functions are concave on $R_{+}^{n}$. When $\bar{x}=(2.2), q(\bar{x})>0$. Also $g(\bar{x})>0$ (here $\hat{x}=\bar{x}$ ). By Theorem 1, an optimum exists, in fact at $(1,0)$.

Example (B) Minimize $x_{1}$ subject to $x_{1}+x_{2} \geqslant 1, x_{1} \geqslant 0, x_{2} \geqslant 0$.

Example (C) Minimize

$$
f(x)=\left\{\begin{array}{ll}
(x-1)^{2}+5 & (x<1), \\
5 & (x \geqslant 1)
\end{array}\right\}
$$

subject to $x \geqslant 0$.

Both (B) and (C) have minima, but no $\bar{x}$ is $q(\bar{x})(=\nabla f(\bar{x})$ for a differentiable problem) strictly positive.

Example (D) Minimize

$$
F(x)=-e^{-x_{1}^{2}-x_{2}^{2}}+\left\{\left[x_{1} x_{2}\right]\left[\begin{array}{ll}
1 & 0 \\
0 & 2
\end{array}\right]\left[\begin{array}{l}
x_{1} \\
x_{2}
\end{array}\right]\right\}^{1 / 2}
$$

subject to

$$
\begin{aligned}
& g_{1}(x)=-\left(x_{1}-1\right)^{2} \geqslant 0, \\
& g_{2}(x)=x_{2}^{3}+1 \geqslant 0, \\
& x_{1} \geqslant 0, \quad x_{2} \geqslant 0 .
\end{aligned}
$$


Here the objective function $F$ is pseudoconvex on the feasible set; $q(\bar{x})>0$ for $\bar{x}=(1,2)$; so a minimum exists, in fact at $(1,0)$.

Example (E) Minimize $F(x)=-e^{-x^{2}}$ subject to $g(x)=x^{3}+x+1 \geqslant 0, x \geqslant 0$.

Since $F$ is pseudoconvex, $g$ is pseudoconcave, and $q(\bar{x})>0$ at $\bar{x}=2$, it follows that there exists an optimal solution; in fact, at $x=0$.

Example (F) Minimize $x_{2}$ subject to $x_{2}-e^{-x_{1}} \geqslant 0, x \geqslant 0$.

If $F$ is convex over $X$ with respect to $X$, and attains its minimum at a unique $x^{0}$, and there exists $z \in X$ with $0 \neq z-x^{0} \geqslant 0$, then $q_{1}(z)>0$, for some $i$; but $q(z)>0$ cannot be deduced. This is shown as follows. Since $f$ is convex, so is $F$. From $F(y)>F\left(x^{0}\right)$ whenever $y \in X, y \neq x^{0}$, there follows

$$
\begin{aligned}
& f\left(x^{0}\right)+\sum_{j=1}^{p}\left(x^{0^{T} B_{j} x^{0}}\right)^{1 / 2}-f(y)-\sum_{j \in J \backslash K(y)}\left(y^{T} B_{j} y\right)^{1 / 2} \\
& -\left\langle q(y), x^{0}-y\right\rangle-\sum_{j \in K(y)}\left[\left(x^{0}-y\right)^{T} B_{j}\left(x^{0}-y\right)\right]^{1 / 2} \geqslant 0 .
\end{aligned}
$$

But $B, y=0$ for $j \in K(y)$ implies that the last summation reduces to $\Sigma_{j \in K(y)}\left(x^{0^{T}} B_{j} x^{0}\right)^{1 / 2}$. Hence $\left\langle q(y), y-x^{0}\right\rangle>0$ for all $y \in X, y \neq x^{0}$. The result follows with $y=z$.

\section{References}

[1] B. C. Eaves, “On quadratic programming," Management Sci. 17 (1971), 698-711.

[2] M. Kojima, "A unification of the existence theorems of the nonlinear complementarity problem," Math. Programming 9 (1975), 257-277.

[3] D. G. Mahajan and M. N. Vartak, "Generalizations of some duality theorems in nonlinear programming," Math. Programming 12 (1977), 293-317.

[4] O. L. Mangasarian, Nonlinear programming (McGraw-Hill, New York, 1969).

[5] B. Mond, "A class of nondifferentiable mathematical programming problems," J. Math. Anal. Appl. 46 (1974), 169-174.

[6] B. Mond and J. Parida, "Existence theory for programming and associated complementarity problems," Pure Mathematics Research Paper No. 81-15, August 1981, La Trobe University, Melbourne, Australia.

[7] B. Mond and $\mathrm{M}$. Schechter, "On a constraint qualification in a nondifferentiable programming problem,' Naval Res. Logist. Quart. 23 (1976), 611-613. 
[8] J. Parida and B. Sahoo, "On the complementarity problem over polyhedral cone," Indian J. Pure Appl. Math. 11 (1980), 838-844.

[9] F. Riesz and B. Sz.-Nagy, Functional analysis (translated from the 2nd French edition by L. F. Boron), (Frederick Ungar Publishing Co., New York, 1955).

[10] C. Singh, "A system of inequalities and nondifferentiable mathematical programming," $J$. Optim. Theory Appl. 27 (1979), 291-299.

[11] S. M. Sinha, "Stochastic programming," Research Report No. 22, Operations Research Center, University of California, Berkeley (1963).

[12] S. M. Sinha, "An extension of a theorem on supports of a convex function," Management Sci. $12(! 966), 380384$. 